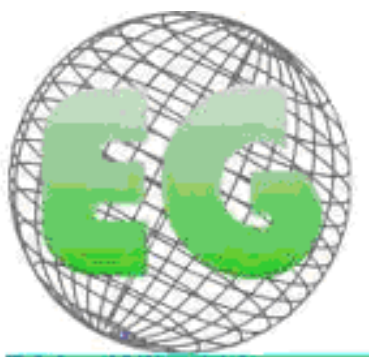

15SN $1696-6$ *⿻

$N^{\circ} 19$

\title{
LACTANCIA MATERNA Y RELACIÓN MATERNO FILIAL EN MADRES ADOLESCENTES
}

BREASTFEEDING AND THE RELATIONSHIP BETWEEN MOTHER AND CHILD IN ADOLESCENT MOTHERS

*Morillo, B., *Montero, L.

*Magíster en Enfermería Materno Infantil. Docente de la de la Escuela de Enfermería Dra. Gladys Román de

Cisneros. Universidad de Carabobo. Valencia. Venezuela.

Palabras clave: Adolescencia, Sexualidad, Lactancia Materna y Vínculo Afectivo.

Keywords: Adolescence, Sexuality, Breastfeeding, and Bonding.

\section{RESUMEN}

Cuando reflexionamos ¿por qué el alto índice de embarazo en adolescentes? ¿por qué el aumento de la desnutrición Infantil?, ¿por qué la mortalidad infantil es tan elevada?, en la búsqueda causal, llegamos a considerar diversos factores que pueden influir en la aparición de estos problemas de salud que afectan a la población infantil y a las/los adolescentes venezolanos, que afectan a su calidad de vida. Uno de los principales factores causales es la trasgresión alimentaría a que son objeto los lactantes y niños pequeños y el abandono de la Lactancia Materna. El presente ensayo destaca la importancia de la alimentación materna exclusiva como función biológica esencial para el ser humano en sus primeros años de existencia humana, así como el visualizar la importancia del amamantamiento en las madres adolescentes y el valor de la relación materno filial. Por otra parte se plantea la necesidad de proporcionar apoyo y orientación a este grupo de madres adolescentes para de esta manera lograr un desarrollo pleno y armonioso de la maternidad, donde se destaque la importancia del cuidado del niño en sus variables: Lactancia Materna y Nutrición, prevención de enfermedades, garantizándoles un correcto crecimiento y desarrollo; así como también la importancia del vínculo afectivo y el correcto desempeño del rol materno por parte de la madre adolescente.

\section{ABSTRACT}

When we ask ourselves: Why the high rate of teenage pregnancy? Why the rise in child malnutrition? Why is infant mortality so high? In a causal search, we consider various factors that may influence the onset of these health problems affecting the child population and of Venezuelan adolescents, affecting their quality of life. One of the main causal factors is the transgression of breastfeeding being given to the infants and toddlers and its abandonment. This article underlines the importance of exclusive breastfeeding as a biological function essential for humans in their first years of existence, and to visualize the importance of breastfeeding for adolescent mothers and the value of mother-child 
relationship. On the other hand, there is the need to provide support and guidance to this group of adolescent mothers and in this way achieve a full and harmonious development of motherhood, wich stresses the importance of child care in its variables: Breastfeeding and nutrition, disease prevention, guaranteeing proper growth and development, and also the importance of proper bonding and maternal role performance by the teen mother.

\section{INTRODUCCIÓN}

La adolescencia es el período del crecimiento y desarrollo humano, que se extiende desde la pubertad, en la que el individuo adquiere la capacidad reproductiva, transita los patrones psicológicos de la niñez a la adultez y consolida la independencia socio - económica; cronológicamente está ubicada entre los 10 y 19 años de edad. Biológicamente la adolescencia es el inicio de la madurez sexual y finaliza socialmente con la independencia del núcleo familiar (1).

En el periodo de la adolescencia se distinguen tres etapas: adolescencia temprana, adolescencia media y adolescencia tardía (2). La primera etapa o la adolescencia temprana, es aquella comprendida desde los 10 - 13 años, considerada biológicamente como el periodo peri puberal, con cambios corporales y funcionales, donde se destaca la menarquía, en las adolescentes de sexo femenino; podemos decir que psicológicamente el adolescente comienza a perder interés por los padres e inicia amistades básicas con individuos del mismo sexo, intelectualmente aumentan sus habilidades cognoscitivas y sus fantasías, no controlan sus impulsos y se plantean metas irreales. Personalmente se preocupa mucho por sus cambios físicos.

La segunda etapa o adolescencia intermedia, correspondiente desde los 14 - 16 años, llamada adolescencia propiamente dicha, en esta etapa el adolescente ha completado su crecimiento y desarrollo somático. Psicológicamente podemos decir que es un periodo de conflicto con sus padres, y por lo tanto busca establecer máxima relación con sus pares. Para muchos es la edad promedio donde se inicia la actividad sexual, se sienten invulnerables y asumen conductas que generan grandes riesgos, ocupan parte de su tiempo preocupados por la apariencia física, pretende tener un cuerpo más atractivo y se manifiestan fascinados con la moda.

En la tercera y última etapa llamada adolescencia tardía, que va desde los 17 a los 19 años, ya casi no se presentan cambios y han aceptado su imagen corporal, se acercan nuevamente a sus padres, desarrollando su propio sistema de valores, donde uno de los aspectos de mayor relevancia durante la adolescencia es la búsqueda de su propia identidad y el logro de la identidad sexual.

El hecho de verse a sí mismo como ser sexual, poseer capacidad de desarrollar una relación intima, romántica, son aspectos importantes del logro de la identidad sexual del adolescente, razón por la cual los/las adolescentes comienzan la búsqueda de su identidad sexual, factor que posteriormente los podría llevar al inicio de relaciones sexuales de forma precoz, aumentando el riesgo de embarazo en esta etapa de la vida, y a su vez trayendo como consecuencia, madres adolescentes no preparadas cognitivamente para enfrentar el nuevo rol (3). 


\section{SEXUALIDAD Y ADOLESCENCIA}

En relación a la sexualidad de los adolescentes, es algo que en muchos casos llega a perturbarlos, debido a que ven coartada su libertad de expresión en este tema, convirtiéndolo en tabú, creándose en los jóvenes grandes vacíos e interrogantes, ocasionando que busquen respuestas en lugares y personas no aconsejables.

Los jóvenes son apasionados y tienden a dejarse llevar por sus impulsos, particularmente los sexuales, son volubles y sus deseos pueden ser inconstantes además de transitorios y vehementes. Todo lo llevan a los extremos, aun tratándose del amor, el odio o cualquier otra cosa, se sienten sabelotodos. Son cajas de sorpresas de todos los colores, formas, tamaños, únicos e irrepetibles, crecen creativos y con incontables cosas para dar y necesitan que los orienten para ser un adulto responsable, donde el desarrollo de la adolescencia esté dirigida y dominada por la urgente necesidad de dar un nuevo sentido al YO.

Por esta razón el embarazo como una de las múltiples consecuencias del inicio temprano de las relaciones sexuales, que experimentan los/las adolescentes, originan situaciones conflictivas, emocionales y sociales que conllevan al rechazo de la maternidad.

Venezuela es considerada como líder en embarazos no deseados donde se señala que de cada mil mujeres 98 tienen entre 15 y 19 años de edad, y que una de cada 10 comienzan su vida sexual antes de los 15 años (4); para el 2002, Venezuela presenta un 21,35\% de nacimientos registrados en menores de 20 años (5). Considerando un importante subregistro, se observa una tendencia sostenida al descenso de $21.77 \%$ (113.476) en 1999 , a $21.40 \%$ (116.516) en 2000 , a $21.10 \%$ (111.769) en 2001 y $21.35(105,210)$ en 2002 . La fecundidad temprana es $51 \%$ mayor en zonas menos urbanizadas en comparación con el área metropolitana de Caracas. Las adolescentes menos instruidas tienen una fecundidad 4 veces más elevada que las más instruidas. El nivel de fecundidad de las venezolanas es similar al de las colombianas, brasileñas, bolivianas y muy inferior al de las centroamericanas (6). La iniciación sexual temprana es mayor en las menos instruidas, 1 de cada 3 ha tenido relaciones sexuales, en las más instruidas esa proporción se reduce a 4\%.

La sexualidad forma parte del desarrollo normal del niño y el adolescente. Desde la infancia el niño experimenta sensaciones placenteras al tocarse, al ser acariciado y besado, observando las expresiones de afecto y actitudes sexuales de los adultos que lo rodean, que en ocasiones tratan de imitar. Durante el período puberal se establece la identidad sexual donde sigue recogiendo información, sobre la sexualidad con los amigos, los maestros y la familia. En esta etapa se pueden desarrollar actitudes negativas hacia la sexualidad si los adultos evitan hablar del reconocimiento de su cuerpo, en relación a su sexualidad, catalogándolas como eufemismos o rechazos, cualquier conducta relacionada con el sexo, incluidas las que el niño puede manifestar espontáneamente como la erección. (7).

La actividad sexual del adolescente suele ser esporádica, con periodos prolongados durante los cuales el adolescente permanece en abstinencia. Muchos adolescentes mantienen lo que se denomina una "monogamia seriada", con enamoramientos intensos y apasionados, pero de duración más corta que la del adulto. Son más frecuentes las disfunciones sexuales, que pueden guardar relación con: temor al embarazo, a ser descubiertos, conflictos de conciencia o actuar bajo presión de la pareja. También influyen las condiciones en que muchos adolescentes mantienen relaciones sexuales, de forma incómoda y rápida (en los asientos de un coche, en un rincón oscuro de una discoteca o de la calle, en los cines, entre otros). 
Si se conjugan ejercicio de la sexualidad y la omnipotencia propia del adolescente, afirmando que jamás les ocurrirá ningún daño, algo parecido ni igual a lo de su amiga, utilizando frases como "eso no me sucederá a mí", de allí que se encuentre en situaciones de riesgo conflictivas y emocionales como lo es el embarazo, y enfrentar la sociedad y asumiendo si lo desea la lactancia materna sin comprender los beneficios que esta representa para su bebé.

Muchas adolescentes llegan al embarazo como una manera de llenar sus carencias, de ser requeridas sexualmente, las hace sentirse valoradas y se entregan a una relación sin que necesariamente sea eso lo que desean realmente. Dan este cariño no tanto por la satisfacción personal sino por satisfacer a la persona que quieren mantener a su lado. (8).

La maternidad es la capacidad pro creativa para la continuación de la especie humana donde se mezclan elementos de orden afectivo, social, económico, psicológico, educativo, ético y cultural, aunque para algunas culturas, la maternidad es factor de crecimiento, mientras que para otros, este proceso se transforma en un problema social, atacándose generalmente el emergente y no profundizando en las causas transformadoras de las adolescentes con el entorno, llámese familia, educación o sociedad.

Desde otro orden de ideas, la adolescencia está permanentemente como temática en la actualidad, como un período turbulento, de hormonas embravecidas, donde los adultos, deben comprender que las/los adolescentes sienten curiosidades, deseos de tener relaciones sexuales, encontrándose con grandes dudas y carencias afectivas, donde la mayor preocupación son los daños irreparables, que pudieran sufrir en relación con otras personas, tales como físicas (I.T.S ), psicológicas (temprana iniciación sexual por presión de grupos, sufrimientos amorosos, embarazos, madres solteras, entre otras).

\section{LACTANCIA MATERNA}

En los últimos años hemos visto un marcado aumento de embarazos en adolescentes entre 14 y 19 años y junto con ello una disminución de la práctica de la Lactancia Materna, la que no alcanza a llegar a los 6 meses de vida de los lactantes. La lactancia materna es considerada la mejor forma de alimentación natural exclusiva para el neonato hasta los 6 meses de edad, siguiendo con la alimentación complementaria más idónea hasta los 2 años, que consiste en incorporar otros alimentos específicos para el lactante, además de constituir un factor de protección celular y humoral, fortaleciendo la afiliación materno filial (9). La lactancia materna la asociamos muy merecidamente con la epidemiología, porque influye en la reducción de la tasa de morbimortalidad infantil, en los indicadores de salud de enfermedades infecciosas, y por otro lado no menos importante, mejora el desarrollo cognitivo.

En 1.989, la O.M.S y la UNICEF publicaron una declaración conjunta para proteger, promover y apoyar la lactancia materna. En el estudio realizado por Kramer y cols. en referencia al crecimiento (10), se sugiere que la lactancia materna prolongada y exclusiva, realmente podría acelerar el crecimiento (incremento de peso y talla) durante los 6 primeros meses de la vida. En un estudio realizado recientemente en España no se aprecian diferencias entre la población amamantada y la población general en cuanto a desarrollo físico (11). En cuanto al crecimiento cerebral expresado por el aumento de la circunferencia craneal, destaca el mayor aumento en los niños amamantados. 
En 1994 la Asamblea de Salud de la OMS solicitó la elaboración de nuevas curvas de crecimiento de los niños amamantados y por ello se puso en marcha el Estudio Metacéntrico de la OMS sobre el Patrón de Crecimiento. Éste se llevó a cabo entre 1997 y 2003 y se centró en la obtención de datos de crecimiento e información de 8.440 lactantes amamantados y niños pequeños de diferentes orígenes étnicos y entornos culturales (Brasil, Estados Unidos de América, Ghana, India, Noruega y Omán). La muestra conjunta de los 6 países participantes ha permitido la elaboración de un patrón verdaderamente internacional. Estas nuevas gráficas se han puesto a disposición de los profesionales en el año 2006 (12).

Este nuevo patrón de crecimiento infantil proporciona por vez primera, datos científicos y orientación sobre el modo que los niños de todo el mundo deberían crecer. Se confirma que todos los niños, nacidos en cualquier parte del mundo, que reciban una atención óptima desde el comienzo de sus vidas, tienen el potencial de desarrollarse en la misma gama de pesos y tallas. Por tanto las diferencias en el crecimiento infantil hasta los cinco años dependen más de la nutrición, las prácticas de alimentación, el medio ambiente y la atención sanitaria que de los factores genéticos o étnicos. Se considera lactancia natural como "norma" biológica y establece el lactante alimentado al pecho como el modelo normativo de crecimiento. Esto asegura por primera vez, la coherencia entre los instrumentos utilizados para evaluar el crecimiento y las directrices nacionales e internacionales sobre alimentación infantil que recomiendan la lactancia materna exclusiva durante 6 meses y posteriormente con alimentos complementarios hasta la edad de 2 o más años. A partir de ahora, se podrán evaluar, valorar y medir con precisión los resultados de la lactancia materna y la alimentación complementaria. No hay duda de que la alimentación materna al lactante constituye una función biológica esencial para el ser humano. Siendo el deseo, que cada madre adolescente en cualquier parte del mundo que desee amamantar a su hijo, tenga la información y el apoyo necesario, pues siendo la lactancia materna, la forma más maravillosa, armoniosa y sencilla de nutrir a su bebé, a través de esas gotitas cargadas de amor, porque al ofrecerle conocimiento y recursos sobre dicho vital líquido a las/los adolescentes, se le proporciona educación básica y práctica sobre los beneficios y las herramientas para el éxito en el amamantamiento en conjunto con su entorno familiar hacia su bebé.

El amamantamiento es una experiencia satisfactoria tanto para la madre como para el hijo por el contacto piel con piel que se produce entre ambos, la madre es extraordinariamente sensible durante la lactancia y su mayor preocupación gira en torno a la alimentación y cuidado de su hijo. Sin embargo, en las madres adolescentes, debido fundamentalmente a la etapa de la vida que están viviendo, al desconocimiento y falta de experiencia esto les provoca "angustia". La madre adolescente es una mujer joven, necesita vivir esa etapa de su vida, ya que la maternidad temprana no la hace mujer adulta; no está preparada, en un corto tiempo debe lograr importantes metas, asumir su sexualidad, su salud reproductiva, auto cuidado, relaciones afectivas con la familia, con su pareja, con su hijo. Son tareas y desafíos que las jóvenes nunca han abordado y llevan a estas madres adolescentes a sentirse recargadas de trabajo y con una carga emocional muy fuerte, lo que constituye un gran riesgo para la Lactancia Materna.

Dar de mamar es el mejor regalo para comenzar la vida de un niño; dar el pecho hace crecer el amor entre la madre y el hijo. El contacto con la piel suave y caliente de la madre le da tranquilidad y placer a ambos. Es el mejor método de alimentación de los lactantes, por razones tanto económica, como higiénica y afectiva. La leche materna es un recurso material de bajo costo, el cual contiene proteínas que favorecen la respuesta inmunitaria y elementos que fomentan la respuesta del cerebro, cabe también destacar que la lactancia natural, refuerza los lazos afectivos de la familia, menos casos de maltrato y abandono del 
niño, reduce el costo de consultas médicas y de medicamentos, exámenes y hospitalizaciones del niño, facilita la alimentación en la noche y en los viajes, madres e hijos más saludables, disminuye el uso de sucedáneos de La leche Materna, disminuye la contaminación ambiental al disminuir los envases de sucedáneos no reciclables.

\section{FACTORES QUE INFLUYEN EN LA PRÁCTICA DE LA LACTANCIA MATERNA.}

* Deficiente información de la madre, el cónyuge y demás familiares. Los conocimientos que tienen un gran número de madres son insuficientes y no pocas veces contrapuestos a la realidad, son fácilmente presas de las absurdas decisiones, por familiares o amigos que ejercen sobre ellas una influencia negativa.

* La falta de confianza de la madre en su capacidad para amamantar a su hijo y producir leche en cantidad suficiente, puede ser grave motivo de preocupación, lo cual a su vez, puede inhibir la bajada temprana de la leche y su flujo normal.

* La leche materna se produce como resultado de la acción conjunta de hormonas y reflejos los cuales pueden ser inhibidos por diversas situaciones emocionales de la madre. Cada vez que el niño succiona, estimula las terminaciones nerviosas del pezón y la areola; estos llevan mensajes a la hipófisis de la madre, que responde produciendo una hormona llamada prolactina; ésta va por la sangre hasta el alvéolo mamario y hace producir la leche. Las terminaciones nerviosas estimuladas mediante la succión del pezón y la areola llevan también un mensaje al cerebro de la madre; donde se produce otra hormona llamada oxitócica; ésta, al igual que la prolactina, va por la sangre hasta los pechos y actúa, produciendo contracción de las células musculares que rodean los alvéolos, impulsando así la salida de la leche a través de los conductos galactóforos hasta el pezón. Este reflejo de la oxitócina, no depende sólo de la succión del pezón, sino también de las emociones y sentimientos de la madre.

* Las preocupaciones frenan el reflejo de la succión. Hay relación de la depresión con disminución del periodo de lactancia natural. La producción de leche depende de la frecuencia con que se le da el pecho al niño, la fuerza de succión del niño, el reflejo de bajada de la leche y el uso de anticonceptivos orales.

* Los cambios en la asistencia del parto y la interferencia hospitalaria: la demora del inicio de la succión del pecho puede traer inconvenientes posteriores para la lactancia. El tetero induce al rechazo de la succión del pezón, ya que esta última requiere un esfuerzo mayor por parte del niño. Aplicar demasiada anestesia durante el parto; mantener un horario fijo y no espontáneo.

* Inconvenientes anatómicos: Pezones invertidos la mayoría de ellos pueden ser modificados por maniobras y masajes. Las fisuras, agrietamientos y posterior infección del pezón son en gran parte prescindibles, evitando el aumento de tensión láctea de los senos.

* Autoimagen y sexualidad: Existe un grupo de mujeres que están dispuestas a sacrificar la lactancia en beneficio de su estética. Muchas mujeres temen que la lactancia arruine la figura del pecho. Muchas mujeres se avergüenzan de amamantar en público.

* Actividad social y trabajo de la madre: Obstáculo considerable tanto para el inicio como para el mantenimiento de la lactancia. Desconocimiento de la madre lactante de la protección legal.

* Nuevos valores sociales: A menudo se considera a la lactancia materna como anticuada, signo de atraso o como una vulgar práctica campesina. El biberón se ha convertido en un signo de posición.

* El papel de la industria, la facilidad de contar con fórmulas cada vez más parecidas física y bioquímicamente a la leche humana ha descendido la práctica de la lactancia 
materna. El impacto de la publicidad comercial contrapone al éxito de la crianza natural el beneficio de la crianza artificial.

* Eliminación de drogas por la leche: Casi todas las sustancias ingeridas por la madre van a aparecer en la leche, existen en la leche materna enzimas que metabolizan a los fármacos (acetilación de las sulfas). Casi nunca es necesario suspender la lactancia materna definitiva debido a que la madre esté tomando una droga. El anticonceptivo común de estrógeno y progesterona disminuye el abastecimiento de leche, mientras que la progesterona sola reduce su contenido proteínico.(13)

\section{VÍNCULO AFECTIVO MADRE/HIJO}

Otro aspecto a considerar en este artículo, que no se puede dejar de mencionar, es lo relacionado con el vínculo afectivo madre/hijo/a cuando una adolescente amamanta. El niño en su desarrollo intenta a través de su comportamiento estar cerca de la madre, utilizando conducta como succionar, sonreír, llorar y aferrarse. Esta búsqueda y necesidad de cercanía de la madre, con respecto al niño, se conoce como conducta de apego y la conducta de la madre que intenta mantener al hijo cerca se denomina conducta de atención. La conducta de ambos, que se dirige a buscar y mantener la proximidad de uno con el otro se llama conducta afectiva (14).

Los seres humanos, al igual que el resto de los animales, nos relacionamos con el mundo que nos rodea a través del sistema nervioso. Este nos permite captar lo que está ocurriendo en él, a través de los órganos de los sentidos, vía sensorial o aferente. Esta información llega a los centros de integración que son grupos de neuronas que procesan esta información y envían su respuesta a través de la vía eferente o motora. El sistema nervioso tiene sus centros de integración más simples en la médula espinal, ahí residen los centros de la micción, defecación, marcha, etc. Subiendo desde la médula hacia arriba, nos encontramos con centros de integración cada vez más complejos. Así tenemos como en la parte central del cerebro se encuentra el paleocórtex lugar donde residen los centros de integración de los instintos. En la parte superior, está la corteza cerebral o neocórtex donde residen los centros de integración de los actos voluntarios, donde se usa la razón para dar una respuesta (actos racionales). Al nacer heredamos en nuestros genes una serie de conductas que se han aprendido a través de la evolución y que heredamos en nuestros genes. Esta es la herencia genética. En cambio el neocórtex es como un libro prácticamente en blanco, que lo vamos a escribir a través del aprendizaje. Esta es la herencia cultural.

La comunicación entre los seres humanos se realiza en más de un 50\% a través de gestos, miradas, actitudes corporales etc. El lenguaje oral participa minoritariamente en la comunicación. $Y$ así podríamos citar una serie de conductas innatas que prácticamente rigen nuestra conducta. En el ser humano puede sobrevivir, pero le traerá muchos problemas de salud física y mental. El formar el vínculo es un aprendizaje (neocórtex). El apego puede variar de una adolescente a otra, de un día a otro, de una hora a otra, dependiendo de variables, como: el hambre, la fatiga, el estado emocional y psicosocial de la adolescente, influyendo la situación específica del momento en el amamantamiento. Siendo importante destacar que es un momento donde debe estar acompañado de profundo sentimiento, donde se estimula el amor en el niño y se vinculan valores que desarrollaran confianza, y de esta conducta de apego, dará lugar a conductas intelectuales, formación de conceptos, el relacionarse con otras personas, es decir madre/ hijo/a, experimentaran una relación afectuosa, íntima y continua en cada mamada en las que ambos sientan satisfacción y gozo, siendo uno de los beneficios importantes en la lactancia materna, con la calidez que se 
proporciona en el amamantamiento, demostrando la seguridad que transmiten los brazos maternos (15).

Las ciencias biológicas nos indican que es la primera hora que sigue del nacimiento la que conforma todo un período crítico que influye en el desarrollar la capacidad de amar. Las investigaciones indican que un vínculo seguro entre la madre y el niño durante la infancia influye en su capacidad para establecer relaciones sanas a lo largo de su vida, cuando los primeros vínculos son fuertes y seguros la persona es capaz de establecer un buen ajuste social, por el contrario la separación emocional con la madre, la ausencia de afecto y cuidado puede provocar en el hijo una personalidad poco afectiva o desinterés social. Según indican estas investigaciones, la baja autoestima, la vulnerabilidad al estrés y los problemas en las relaciones sociales están asociados con vínculos poco sólidos. Si las experiencias de vínculo han sido negativas y graves, el ser humano es más propenso a desarrollar trastornos psicopatológicos. Son las interacciones madre-niño las que influyen en el desarrollo socio-emocional $y$ en la conducta actual y futura del menor.

Han sido numerosos los estudios realizados que verifican que el desarrollo del cerebro está directamente vinculado a ser afectado por las relaciones tempranas del bebé, que establece con sus padres, ya que podrían ser las bases a procurar y recibir atenciones y cuidados de otros. La creación del vínculo afectivo es una experiencia maravillosas mediante la cual, el bebé y la madre aprenden a amarse mutuamente, y comienza desde la primera mirada percibida por ambos, luego del parto, fortaleciéndose con el tiempo, siendo determinante para el desarrollo psicomotor y social del bebé. A través de la conversación, el contacto, el tacto y en busca del apoyo materno, donde los primeros años de vida, tanto del hijo como el de la madre, serán para ambos una experiencia de aprendizaje.

\section{SER UNA MADRE ADOLESCENTE}

Las condiciones de una mujer para generar un buen "apego" se van produciendo desde su infancia, influyen positivamente los juegos con muñecas, la visualización de un embarazo de su madre, hermana mayor o pariente. Luego cuando adulta, son importantes: la planificación, confirmación y aceptación del embarazo, condiciones que no se dan en las madres adolescentes. Estas madres adolescentes se encuentran en un período de cambios biológicos sicológicos y sociales, en un período de búsqueda de ¿Quién soy?, en donde se definen los rasgos de la personalidad; existe inestabilidad emocional, inmadurez y donde un grupo de amigos es lo más importante. En este período gustan de fiestas, de compartir con otros, lo que al tener un hijo se ve frustrado, pues el bebé necesita cuidados especiales durante los primeros años de su vida. Además, muchas veces los hijos no son deseados, cuestión fundamental para amamantar, criar y educar a un hijo.

Estas razones nos explican en cierta medida el porqué muchas madres adolescentes no logran Lactancia Materna exclusiva seis meses. Los hijos de madres adolescentes tienen mayor riego de nacer con bajo peso, según las circunstancias en que se haya llevado a cabo el embarazo. Es frecuente el riesgo de estos niños a ser sometidos a abusos físicos, negligencia en los cuidados de salud, alimentación y desarrollo físico y emocional, por la incapacidad de la madre para discriminarse con el bebé. Ellas priorizan sus demandas sobre las necesidades del niño por ser todavía demandantes por su corta edad, esto hace que no tengan la capacidad de contener al hijo. Tienen poca tolerancia a la frustración, les cuesta entender que el hijo no es como ella quiere, ni hace lo que ella desearía en ese momento, muchas veces esto le produce enojo con el niño, con consecuencia que podría ponerlo en riesgo. Debemos orientar nuestros esfuerzos a las necesidades de salud más desatendidas y apremiantes (15). 
Debemos ofrecer a los adolescentes y a sus padres un asesoramiento sanitario dirigido a la salud integral, mediante la detección de problemas físicos y emocionales en etapas precoces, guías que prevengan las conductas de riesgo y ofrecer programas preventivos para estimular conductas que favorezcan estilos de vida saludables en la edad adulta.

\section{REFERENCIAS BIBLIOGRÁFICAS}

1. Calderón, Y. Prácticas de auto cuidado de salud, en relación con la aparición de infecciones de transmisión sexual en las adolescentes embarazadas. PASAE. Estado Carabobo. (2005).

2. Isler, J. Embarazo en la adolescencia. Revista de postgrado de la cátedra VI de medicina. Nro. 107. (2001).

3. Papalia, D. Desarrollo Humano. Sexta Edición. McGRAW - HILL. Editora: Martha Edna Suárez. México. (1998).

4. Gómez, L. "Conferencia Internacional De Derechos De La Niñez Y Adolescencia" Universidad Central de Venezuela, Ministerio de Salud y Desarrollo Social. (2006).

5. MPPS/OMS/OPS. Población De Adolescentes En Venezuela http://www.monografias.com/trabajos34/pasantias/pasantias.shtml). (2006).

6. ENPOFAM www.sisov.mpd.gob.ve/estudios/15/mortalidad.doc - / www.undg.org/archive_docs/1547-Venezuela_CCA_-_Venezuela_2001.doc-(1998).

7. Anales Venezolanos de Nutriciòn. Vol. 12-14. Editor Fundaciòn Cavendes 2007

8. Pinto. L. F Apuntes Del Curso De Lactancia Materna Puerto. Montt. (2003).

9. Klaus. M La Relación Madre-Hijo Editorial. Panamericana. (1978).

10. Kramer. M. S Biology Bias? Pediatric. 343-347 Probit Study Group. Vol. 110 No. 2 (2002).

11. Galbe. J Los Niños Amamantados De Forma Exclusiva. Revista Pediátrica de Atención Primaria VII 33-41 (2005).

12. The Who Multicentri Growth Reference Study http:www.who.int/childgrowth/mgs/es/.

13. Maritza Landaeta de J. Educación en Nutrición. Vol. 8. Editor Fundacion Cavendes. (2008).

14. Modulo I. Tendencia en Salud Familiar y Comunitaria y Promoción. La Serena. Editor. Centro Latinoamericano de Demografía. (2005).

15. Revista Latinoamericana de Psicologìa. Relaciòn de apego en madres adolescentes y sus bebès canguros. Año/vol. 38, nùmero 001. Fundaciòn Universitaria Konrad Lorenz, Bogotá. 